\title{
Note sur la valeur bromatologique des graminées des "savanes noyées" en Guyane française.
}

\author{
par M. HIDIROGLOU
}

La Guyane française, située en région équatoriale d'Amérique du Sud entre $2^{\circ}$ et $4^{\circ}$ de latitude nord comprend deux régions distinctes:

- la région côtière atlantique, ou Terres basses, de 50 kilomètres de profondeur et 350 kilomètres de longueur:
- le reste du pays, les Terres hautes, couvert par la forêt.

Les Terres basses sont des terres alluviales, pro-égées de l'influence océanique directe par un :ideau de palétuviers. C'est la région des savanes su prairies naturelles sur lesquelles se fait l'éle-

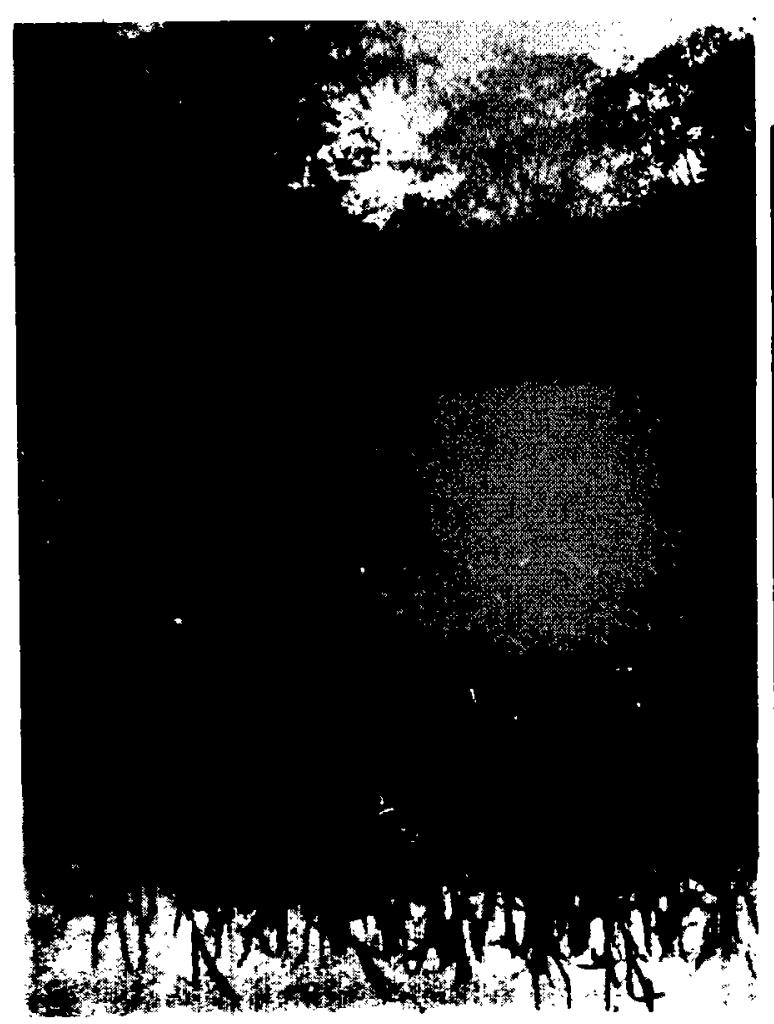

1. Sarane à Panicum Carbininde

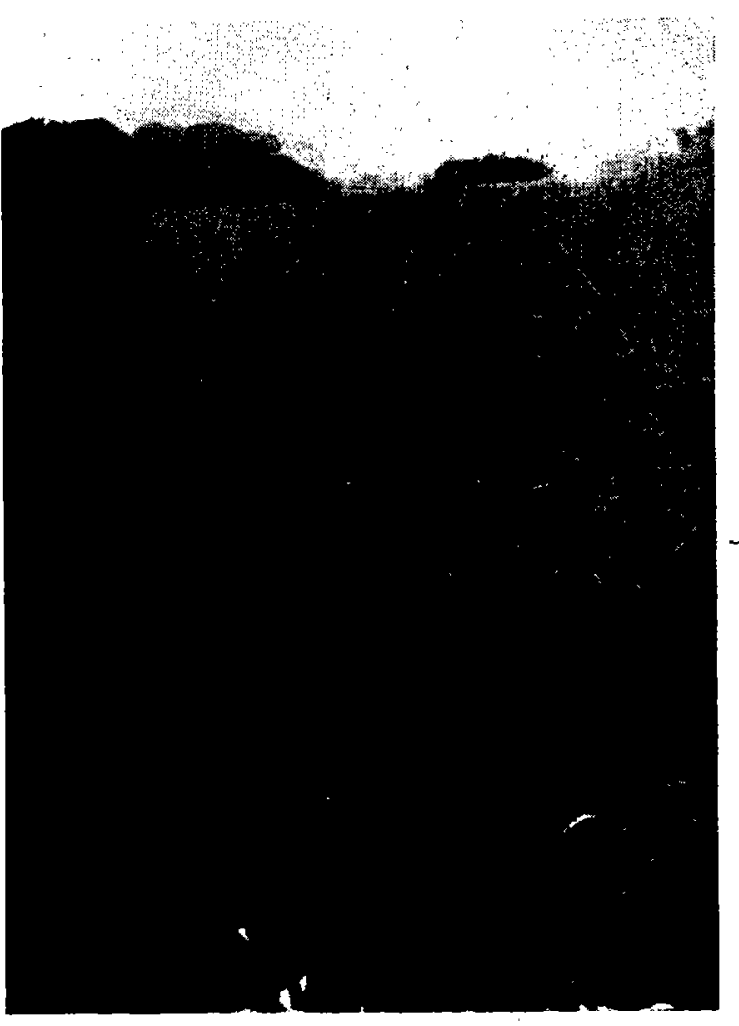

2. Savane à Echinochloa polystachia 
vage. Mais très souvent elles sont marécageuses, noyées en saison des pluies. La mise en valeur de ces « savanes noyées », particulièrement dans le sud-est, est envisagée pour un proche avenir, ce qui permettrait de développer l'élevage.
Le tableau $V$ indique les taux de matières minérales de deux graminées, Echinochloa polystachia et Leersia hexandra.

On pourrait considérer comme pâturages de première qualité dans ces savanes ceux qui renferment plus de 30 p. 100 de Panicum bar-

TABLEAU I - COMPOSITION PHYSIQUE DU SOI

(en pourcentage de terre séchée à l'air)

\begin{tabular}{|c|c|c|c|c|c|c|}
\hline Frofondeur & Terre fine & Argile & total & fin & Iimon & Humidité \\
\hline $\begin{array}{c}10-40 \mathrm{~cm} \\
\vdots\end{array}$ & 100 & 62,8 & 0,7 & 0,7 & 31,5 & 3,8 \\
\hline $50-90 \mathrm{~cm}$ & 100 & 59 & 0,4 & 0,4 & 34,5 & 4,6 \\
\hline $190-200 \mathrm{~cm}$ & 100 & 60 & 0,6 & 0,6 & 33. & 3,1 \\
\hline
\end{tabular}

TABLEAU II - ANALYSE CHIIIQUE DU SOI

(en pourcentage de terre séchée à $105^{\circ}$ )

\begin{tabular}{|c|c|c|c|c|c|c|c|c|}
\hline \multirow{2}{*}{$\begin{array}{l}\text { Pro- } \\
\text { fondeur } \\
\text { fen an }\end{array}$} & \multicolumn{4}{|c|}{$\begin{array}{c}\text { Bases échangeables } \\
\text { en mb } \%\end{array}$} & \multirow{2}{*}{$\begin{array}{c}S \\
\therefore g \\
g\end{array}$} & \multirow{2}{*}{$\begin{array}{c}t \\
\text { mg } \text { So }^{\prime}\end{array}$} & \multirow{2}{*}{$\begin{array}{c}V \\
\text { meg } \%\end{array}$} & \multirow[t]{2}{*}{$\mathrm{Fh}$} \\
\hline & $\mathrm{Cg}$ & $\mathrm{M}_{E}$ & $\mathrm{~K}$ & $\mathrm{Na}$ & & & & \\
\hline & 3 & 13 & 0,45 & 0,8 & 17,4 & 23,4 & 74,3 & 5,4 \\
\hline $190-200$ & 5,2 & 15,2 & 0,72 & 16 & 22,1 & 25,4 & 87 & 7,1 \\
\hline
\end{tabular}

\section{Composition du sol des savanes noyées.}

Ill s'agit d'argiles marines quaternaires dont la composition physique est donnée par le tableau I* ef l'analyse chimique par les tableaüx $11^{*}$ ef $1 \mathrm{H}:$

Valeur nutritive de la végétation des savanes nòyées.

Les herbes les plus couramment rencontrées, dont le tableau IV donne l'ánalyse, sont : Panicüm barbinode, Echinochloa polystachia, Leersia hexandra, Hymenacnae amplexicaulis, Panicum mertenseii, Paspalum riparium; Acroceros zizanoides, Luzeola sp., Cyperus.

* Les tableaux 1 , Il ef Ill nous ont été communiqués par M. Ziefferman, pédologue.
TABLEAU III - ANALYSE CEIMIQUE DU SOL

(pour $100 \mathrm{~g}$ de terre séchée à 1050)

\begin{tabular}{|c|c|c|c|c|c|c|c|c|}
\hline \multirow{2}{*}{$\begin{array}{l}\text { Pro- } \\
\text { fondeur } \\
\text { en cm }\end{array}$} & \multicolumn{4}{|c|}{ Bases totales } & \multirow{2}{*}{$\begin{array}{c}\mathrm{P}_{2} \mathrm{O}_{5} \\
\text { mg }\end{array}$} & \multirow{2}{*}{$\begin{array}{l}C \\
E x\end{array}$} & \multirow{2}{*}{ m } & \multirow{2}{*}{$\frac{C}{N}$} \\
\hline & $\mathrm{Ca}$ & Mg & $\mathrm{K}$ & $\mathrm{Ne}$ & & & & \\
\hline $10-40$ & 8,03 & 53 & 39 & 1,9 & 206,3 & 0,5 & 0,08 & 6,25 \\
\hline $50-90$ & 15,6 & 50 & 45 & 2,7 & 118,1 & & t. & \\
\hline $190-200$ & 15,36 & 68 & 50 & 50 & 149 & & & \\
\hline
\end{tabular}

binode, d'Echinochloa polystachia ou encore de Paspalum riparium, comme pâturages de deuxième qualité ceux qui renferment entre 15 et 30 p. 100 de ces mêmes graminées et comme pâturages de troisième qualité ceux qui en renferment moins de 15 p. 100.

D'après les résultats des analyses, nous pouvons adopter la classification suivante en ce qui concerne le « degré de qualité » de ces plantes:

I. Excellentes:

Panicum barbinode.

Hymenacnae amplexicaulis. 
TABIEAU IV

AUALTE EFFECTUE WII POUTCEFAGE DE MATIEPE SECHE

\begin{tabular}{|c|c|c|c|c|c|c|c|c|c|c|c|c|c|c|c|}
\hline Espricos & anlioité & $\begin{array}{l}\text { metiores } \\
\text { richos }\end{array}$ & Cindres & $\begin{array}{l}\text { Watioros } \\
\text { protúiq. }\end{array}$ & $\begin{array}{l}\text { intiures } \\
\text { motéin. } \\
\text { digest. }\end{array}$ & $\begin{array}{l}\text { Watières } \\
\text { grasses }\end{array}$ & $\begin{array}{l}\text { Viatières } \\
\text { grasses } \\
\text { digest. }\end{array}$ & $\begin{array}{l}\text { Cellu- } \\
\text { lose }\end{array}$ & $\begin{array}{l}\text { Celi11- } \\
\text { lose } \\
\text { digest. }\end{array}$ & $\begin{array}{c}\text { Fxtrnct. } \\
\text { non } \\
\text { azoté }\end{array}$ & $\begin{array}{c}\text { Extract. } \\
\text { non azoté } \\
\text { digest. }\end{array}$ & Glucides & $\begin{array}{l}\text { Eléments } \\
\text { digest. } \\
\text { totaux }\end{array}$ & P & $\mathrm{Ca}$ \\
\hline $\begin{array}{l}\text { Tuniciail } \\
\text { barbino:lo. }\end{array}$ & 87,4 & 12,6 & 6,8 & 14 & 8,4 & 2,2 & 1,12 & 22 & 14,74 & 53,3 & 35,71 & 18 & 61,42 & & \\
\hline Whinoc!ners & 91,5 & $18,5^{2}$ & 7,3 & 7,5 & 4,27 & 2 & 1,2 & 17 & 10,2 & 66,2 & 35,08 & 29 & 52,31 & & \\
\hline $\begin{array}{l}\text { letroia } \\
\text { huxandrat }\end{array}$ & 68,6 & 31,4 & 9,7 & 8 & 3,36 & 2,3 & 0,73 & 25 & 14,25 & 55 & 38,5 & 12 & 57,78 & 0,13 & 0,25 \\
\hline Honcnacnale & 77,6 & 22,4 & 6,3 & 5,7 & 3,27 & 1,9 & 1,09 & 24 & 14.4 & 69,5 & 35,67 & 26 & 56,14 & 0,11 & 0,15 \\
\hline Panictu & 90,6 & 10,4 & 6,4 & 21,5 & 15,2 & 2,2 & 0,96 & 21 & 12,96 & 45,1 & 22,5 & 18 & 51,28 & 0,30 & 0,16 \\
\hline laspllum & 8 & 162 & 8.5 & 8.8 & 6.96 & 2,1 & 1,02 & 22 & 13,02 & 58,6 & 34,69 & 23 & 57,01 & 0,14 & 0,22 \\
\hline ripnrium & 10 & 10,4 & 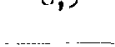 & & & & & & & & . & $\ldots$ & $\cdots$ & & \\
\hline $\begin{array}{l}\text { Acroccros } \\
\text { zizanoities }\end{array}$ & 70,1 & 29,9 & 9,5 & 6,2 & 3,61 & 2,4 & 1,29 & 23 & 13,40 & 58,9 & 34,33 & 33 & 54,14 & 0,16 & 0,22 \\
\hline buzcola $\mathrm{sp}$. & 65,6 & 36,4 & 6,8 & 7,6 & 4,5 & 2 & 1,18 & 26 & 12,48 & 57,6 & 32,14 & 22 & 51,83 & 0,08 & 0,16 \\
\hline Cyncrus & 74 & 26 & 8,2 & 4,8 & 2,72 & 2,4 & 1,15 & 25 & 14,15 & $5 \overline{9,6}$ & 29,8 & 21 & 49,31 & 0,09 & 0,018 \\
\hline
\end{tabular}


WABLEAU

Trux des matières minérales de : E. polystachia et L. hexandra *

\begin{tabular}{|c|c|c|c|c|c|}
\hline \multicolumn{6}{|c|}{ Fésliltats en g pour $100 \mathrm{~g}$ de matière séchée (étuve 105\%) } \\
\hline \multirow{2}{*}{$\begin{array}{c}\text { Eléments } \\
\vdots\end{array}$} & \multicolumn{2}{|c|}{ E. polystachia } & \multirow{2}{*}{$\frac{\text { L. hoxnndre }}{\text { feuilles }}$} & \multirow{2}{*}{$\begin{array}{c}\text { Hótiodes } \\
\text { d'analyses }\end{array}$} & \multirow{2}{*}{$\begin{array}{l}\text { Analyse du } \\
\text { sol } \\
\text { B. } 201\end{array}$} \\
\hline & feuilles & tiges & & & \\
\hline $\mathrm{Ca}$ & 0,21 & 0,18 & 0,19 & & \\
\hline$M_{B}$ & 0,22 & 0,24 & 0,16 & & \\
\hline K & 1,32 & 2,37 & 1,62 & totaux a grès & - Dases \\
\hline $\mathrm{Na}$ & 0,02 & 0,07 & 0,04 & & échan- \\
\hline$P$ & 0,14 & & & et & geables \\
\hline $\mathrm{so}^{4}$ & 0,55 & 2,3 & 2,1 & reprises & \\
\hline $\mathrm{SiO}^{2}$ & 3,56 & 1,85 & 7,32 & aux acides & \\
\hline \multicolumn{5}{|c|}{$\begin{array}{l}\text { Résultats on mg pour } 100 \mathrm{~g} \text { de matiere séchée (étuve 1050) } \\
:\end{array}$} & $\begin{array}{c}\text { mg p. } 100 \text { g avec } \\
\text { acide citrique } \\
25 \% \text { oo }\end{array}$ \\
\hline $\mathrm{Fe}$ & 43 & & & Filéments & 4,2 \\
\hline $\mathrm{cu}$ & 2,2 & & & totawx aorès & 0,13 \\
\hline$M n$ & 9,5 & & 7,5 & calcination & 65 \\
\hline$A I$ & 20 & & & et & 7 \\
\hline $2 n$ & 7,2 & & 10,3 & fusion & 0,67 \\
\hline Mo & 1,75 & & 0,08 & 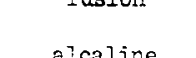 & 0,0008 \\
\hline Co & 1,2 & & 0,02 & alcanine & 0,01 \\
\hline $\mathrm{Cr}$ & 0,1 & & 0,6 & & 0,024 \\
\hline $\mathrm{Ni}$ & 0,22 & & 1 & & 0,024 \\
\hline $\mathrm{Ti}$ & 1,3 & & 0,6 & environ & 0,075 \\
\hline $\mathrm{Sn}$ & 0,07 & & 0,2 & & 0,075 \\
\hline $\mathrm{Pb}$ & 0,22 & & - & & 0,236 \\
\hline
\end{tabular}

2. Bonnes:

Paspalum riparium.

Echinochloa polystachia.

3. Moyennes:

Les autres graminées du tableau IV.

4. Mauvaises :

* Analyse faite au laboratoire de I'Institut d'enseignement et de recherches tropicales, Bondy (Seine).
Les Cyperus.

\section{Evaluation de la production des herbes.}

L'herbe a été recueillie par fauchage des pâtures, les prélèvements se faisant toujours le matin à 8 heures.

Les résultats ci-après on't été obtenus, par mètre carró : 


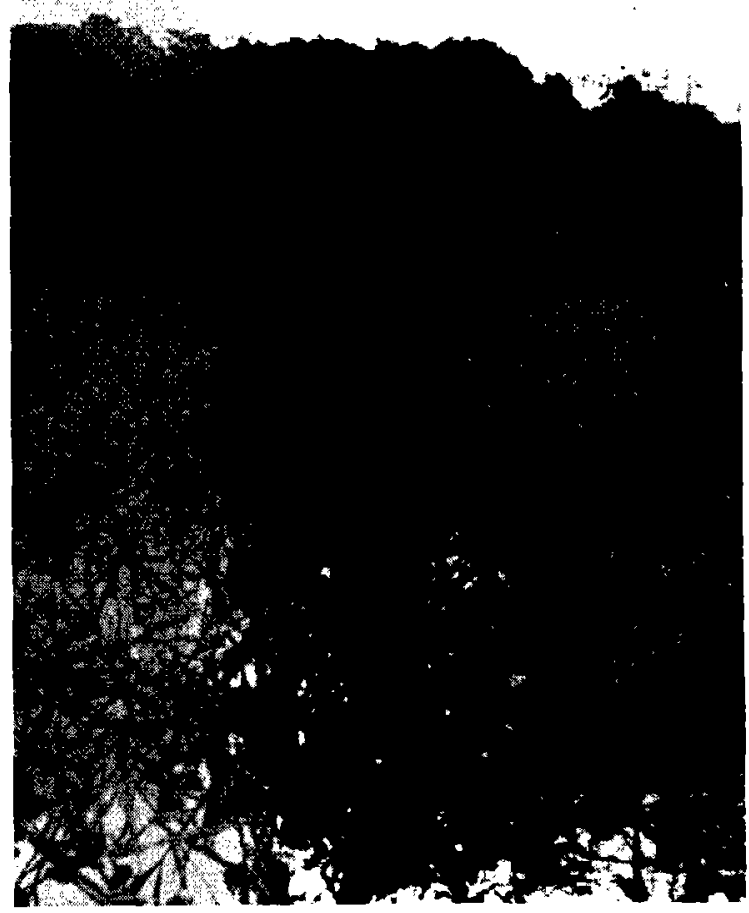

3. Savane d̀ Echinochloa, Para et Paspalum niparium

$I^{\circ}$ Panicum barbinode $=1,800 \mathrm{~kg}$ d'herbe fraîche à 80 p. 100 d'humidité.

$2^{\circ}$ Echinochloo polystachia $=2,200 \mathrm{~kg}$ d'herbe fraîche à 80,1 p. 100 d'humidité.

3" Paspalum riparium $=1,100 \mathrm{~kg}$ d'herbe fraíche à 78 p. 100 d'humidité.

Nous avons dénombré 200 tiges par mètre carré de Panicum barbinode de $60 \mathrm{~cm}$ de hauteur, et il a fallu cing semaines à l'herbe fauchée pour atteindre de nouveau cette hauteur.

\section{Conclusion.}

Ces savanes noyées présentent un intérêt capiia: pour le développement de l'élevage, bubalin en particulier. En effet, pour un pays tel que la Guyane, qui est un des rares pays de l'Amérique q:i puisie compter des troupeaux de buffles, on doit prévoir l'utilisation intensive de ces «savanes royées » en vue de constituer des troupeaux ae buffles hautement sélectionnés pour la production ae lait et de viande, et qui pourraient servir par la suite, de noyau d'approvisionnemert de ce genre d'élevage pour les autres pays je 'Amérique tropicale.

\section{SUMMARY}

Note on the nutrition value of grasses of the swamps of French Guiana

A large part of the iow lying areas of French Guiana is swampy savannah inundated in the rainy season. It is proposed that the area should be put into use. The author records the nature of 
the soil and the grasses found thereon and considers that intensive utilisation could be achieved by the development of buffalo-breeding in these swampy areas.

\section{RESUMEN}

Nota sobre el valor bromatologico de las gramíneas de las « savanas ahogadas »

$$
\text { en la Guayana Francesa }
$$

Una gran parte de las tierras bajas de la Guayana esta constituida por savanas pantanosas, inundadas durante la estación de las lluvias y que deben revalorizarse en un futuro proximo. El autor señala cual es la composicón de este suelo y cuales son las hierbas que en el se encuentran. El cree que la utilización intensiva de esas savanas devería permitir el desarrollo de la cria de búfalos. 